\title{
Quality of Life 1 Year After Laparoscopic Sleeve Gastrectomy Versus Laparoscopic Roux-en-Y Gastric Bypass: a Randomized Controlled Trial Focusing on Gastroesophageal Reflux Disease
}

\author{
L. Ulas Biter ${ }^{1}$ • Michiel M. A. van Buuren ${ }^{1}$ (D) • Guido H. H. Mannaerts ${ }^{2}$ - Jan A. Apers ${ }^{1}$ • \\ Martin Dunkelgrün ${ }^{1}$ - Guy H. E. J. Vijgen ${ }^{1}$
}

(C) Springer Science+Business Media New York 2017

\begin{abstract}
Introduction Bariatric surgery is the only treatment option that achieves sustained weight loss in obese patients and that also has positive effects on obesity-related comorbidities. Laparoscopic sleeve gastrectomy (LSG) seems to achieve equal weight loss as laparoscopic Roux-en-Y gastric bypass (LRYGB), but there is still much debate about the quality of life (QOL) after LSG, mainly concerning the association with gastroesophageal reflux. Our hypothesis is that QOL after LSG is comparable with QOL after LRYGB.

Materials and Methods Between February 2013 and February 2014, 150 patients were randomized to undergo
\end{abstract}

L. Ulas Biter and Michiel M. A. van Buuren are co-first authors.

Michiel M. A. van Buuren

M.Buuren2@franciscus.nl

L. Ulas Biter

u.biter@ franciscus.nl

Guido H. H. Mannaerts

gmannaerts@seha.ae

Jan A. Apers

j.apers@franciscus.nl

Martin Dunkelgrün

m.dunkelgrun@franciscus.nl

Guy H. E. J. Vijgen

g.vijgen@ franciscus.nl

1 Department of Surgery, Franciscus Gasthuis, Kleiweg 500, 3045 PM Rotterdam, Netherlands

2 Department of Surgery, Tawam Hospital Johns Hopkins, 15258 Al Ain, United Arab Emirates either LSG or LRYGB in our clinic. Differences in QOL were compared between groups by using multiple QOL questionnaires at follow-up moments preoperatively and 2 and 12 months after surgery.

Results After 12 months of follow-up, 128 patients had returned the questionnaires. Most QOL questionnaires showed significant improvement in scores between the preoperative moment and after 12 months of follow-up. The Gastroesophageal Reflux Disease Questionnaire (GerdQ) score deteriorated in the LSG group after 2 months, but recovered again after 12 months. After 2 months of follow-up, the mean GerdQ score was $6.95 \pm 2.14$ in the LSG group versus $5.50 \pm 1.49$ in the LRYGB group $(p<0.001)$. After 1 year, the mean GerdQ score was $6.63 \pm 2.26$ in the LSG group and $5.60 \pm 1.07$ in the LRYGB group $(p=0.001)$.

Conclusion This randomized controlled trial shows that patients who underwent LSG have significantly higher GerdQ scores at both 2 and 12 months postoperatively than patients who underwent LRYGB, whereas overall QOL did not differ significantly.

Keywords Bariatric surgery · Sleeve $\cdot$ Bypass · Quality of life $\cdot$ Gastroesophageal reflux disease

\section{Introduction}

The prevalence of overweight and obesity has been increasing over the last 30 years. As of $2015,50 \%$ of adults in the Netherlands are overweight, with almost $14 \%$ being obese [1]. Obesity is associated with significant morbidity, including cardiovascular disease, type 2 diabetes mellitus (T2DM), and mortality [2]. Another frequently seen comorbidity is gastroesophageal reflux disease (GERD) [3]. 
Bariatric surgery is considered to be the best treatment option for patients with morbid obesity in terms of sustained weight loss [4-6]. Not only does bariatric surgery achieve significant excess weight loss, but it also has positive effects on obesity-related comorbidities such as T2DM, hypertension, dyslipidemia, asthma, and sleep apnea [7-9].

The most frequently performed bariatric procedure in Europe is laparoscopic Roux-en-Y gastric bypass (LRYGB). It is regarded as the gold standard in bariatric surgery $[10,11]$. Over the past decade, laparoscopic sleeve gastrectomy (LSG) has gained ground as a new bariatric procedure [11]. In 2013,37\% of the performed bariatric procedures worldwide were LSGs, compared to $28 \%$ in $2011[11,12]$.

LSG is a safe procedure that has multiple advantages. LSG has the technical advantage that mobilization of the small intestine is not needed. Consequently, there is also no possibility of internal herniation, a risk after LRYGB. Due to the intact pylorus muscle, there is a reduced risk of dumping syndrome, and as there is no bypass of the small intestine, we see less malabsorption syndromes in LSG [13]. Lastly, the duodenum is still accessible for endoscopy and interventions such as endoscopic retrograde cholangiopancreatography (ERCP) [14-16]. LSG can also be converted into LRYGB or biliopancreatic diversion with duodenal switch (BPD-DS) if the treatment fails or if complications occur.

According to a recent systematic review, the most frequently reported disadvantage is the onset of GERD after LSG, or worsening of pre-existing GERD. The impact of this complication is controversial however, since the described studies have reported paradoxical outcomes [17]. Several RCTs comparing LSG with LRYGB have been published so far, but these studies have mostly focused on the percentage of excess weight loss (\%EWL) and less on quality of life (QOL). In these studies, the \%EWL appeared to be equal for both LRYGB and LSG [18-26]. Prior concerns were that the gastric sleeve would lose its effect over time, resulting in a decline in weight loss or even weight regain, and the need to convert to LRYGB or BPD-DS [25, 26]. Other long-term studies have disproved these concerns however, since overall QOL appears to be similar after LSG compared with LRYGB [27]. However, most studies have used only general QOL questionnaires and did not focus on specific obesity-related comorbidities such as GERD, which is one of the most important possible complications of LSG [18, 28-31]. Therefore, the aim of this trial is to assess short-term and medium-term differences in QOL between LSG and LRYGB using multiple general and obesity-related QOL questionnaires. Our hypothesis is that QOL regarding GERD and other abdominal symptoms after LSG is comparable with QOL after LRYGB.

\section{Materials and Methods}

\section{Study Design}

This report is based on a randomized controlled trial of patients in our center which had an indication for bariatric surgery. Patients were 1:1 randomized to either LSG or LRYGB using a randomization website. At randomization, patients were stratified for sex, presence of T2DM, and body mass index $(\mathrm{BMI})>50 \mathrm{~kg} / \mathrm{m}^{2}$.

\section{Patient Selection}

All morbidly obese patients who have been approved by the preoperative multidisciplinary team between February 2013 and February 2014 were included in this trial. There was no power analysis, because there were no previous data about these questionnaires in this specific population at the time. The inclusion criteria of this trial are identical to the IFSO criteria for undergoing bariatric surgery [32].

Exclusion criteria were a history of GERD (defined as a gastroscopy with symptoms of GERD, or the use of proton pump inhibitors with symptoms of pyrosis, heartburn, or regurgitation during the preoperative consultation), a symptomatic hiatal hernia [33], severe sweet eating [34], prior bariatric surgery, prior major abdominal surgery, the inability to read or understand Dutch questionnaires, a history of psychiatric and psychological disorders, and unwillingness to be randomized to treatment.

\section{Treatment}

LSG is performed by mobilizing the greater curvature and the posterior wall of the stomach, after which a 34-French (Fr) calibration tube is advanced into the antrum and used as reference. Stapling starts $4 \mathrm{~cm}$ proximal to the pylorus. The used technique was described in 2012 and proven to be effective and safe $[35,36]$.

LRYGB is performed with the antecolic linear technique. A small 4-cm-long pouch is calibrated over a 34-Fr calibration tube, and a $3-\mathrm{cm}$ linear gastroenterostomy is realized. The measured biliopancreatic limb is $60 \mathrm{~cm}$, and the alimentary limb is $150 \mathrm{~cm}$. The omentum can be divided at the surgeon's discretion. Both mesenteric defects are closed with an endoscopic clipping device [35].

All participating surgeons are experienced in bariatric surgery and have performed at least 150 LSGs and 150 LRYGBs. They work in our bariatric center of excellence that performs over 1000 bariatric surgeries per year. The surgeons are allowed to convert LSG into LRYGB and vice versa during surgery by medical indication. Statistical analyses were carried out according to the intention-to-treat principle. 
All patients who undergo bariatric surgery at our center are treated according to a fast-track protocol and are discharged 1 day after surgery if no complications occur [37]. All patients are also prescribed proton pump inhibitors (PPIs) for 6 weeks after surgery. Postoperative follow-up occurs according to the standard of care. Complications are scored at every consultation using the Clavien-Dindo classification [38].

\section{Outcome Measures}

The primary endpoint of this trial is QOL. Multiple questionnaires are filled out to evaluate QOL preoperatively and at postoperative follow-up visits at 2 and 12 months. QOL related to the gastrointestinal tract was assessed using the Gastrointestinal Quality of Life Index (GIQLI), the Bariatric Analysis and Reporting Outcome System (BAROS), and the Gastroesophageal Reflux Disease Questionnaire (GerdQ) [39-41]. The presence of asthma complaints was objectified with the Asthma Control Questionnaire (ACQ) [42]. General QOL was assessed using the EuroQol 5 Dimensions selfreport questionnaire (EQ-5D) and the Short Form (36) Health Survey (SF-36) [43, 44]. Lastly, the Dutch Sweet Eating Questionnaire (DSEQ) was used to evaluate the amount of carbohydrates consumed [34].

Secondary endpoints in this trial were BMI and \%EWL. Since this trial was not powered to detect differences in weight loss between the groups, these data will only be used to assess if BMI and \%EWL are associated with changes in QOL.

\section{Statistics}

Data were analyzed using SPSS Statistics for Macintosh (version 22.0; IBM, Armonk, NY). Values are reported as

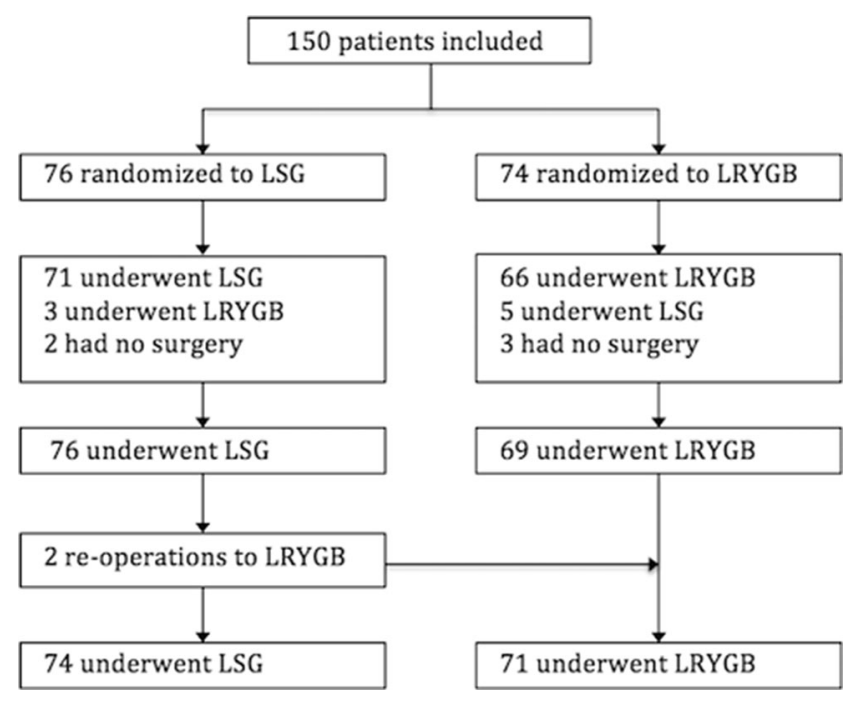

Fig. 1 Flowchart of patient inclusion and received treatment. $L S G$ laparoscopic sleeve gastrectomy, $L R Y G B$ laparoscopic Roux-en-Y gastric bypass

mean \pm SD or mean differences. Descriptive statistics were used for demographics and presence of comorbidities. Scores at each follow-up moment were compared within each group, to assess improvement after surgery, by using Wilcoxon's signed rank test. Scores of each questionnaire were also compared between groups at each follow-up moment by using the Mann-Whitney $U$ test. A two-tailed $p$ value $<0.05$ was considered statistically significant.

Because our sample consists of a relatively small number of patients, listwise deletion of cases with missing values could soon lead to a decrease in statistical power. Therefore, we created a copy of our dataset and used single imputation to increase power and reduce potential bias. With single imputation, missing values were substituted by the median or mode

Table 1 Baseline characteristics

\begin{tabular}{lccc}
\hline & LSG $(n=76)$ & LRYGB $(n=74)$ & $p$ value $^{\mathrm{a}}$ \\
\hline Age $($ mean \pm SD) & $45.5( \pm 11.2)$ & $43.8( \pm 10.8)$ & 0.334 \\
Female sex & $63(82.9 \%)$ & $61(82.4 \%)$ & 1.000 \\
BMI $($ mean \pm SD) & $44.17( \pm 5.00)$ & $44.03( \pm 5.28)(n=73)$ & 0.860 \\
BMI $>50 \mathrm{~kg} / \mathrm{m}^{2}$ & $13(17.1 \%)$ & $13(17.6 \%)$ & 1.000 \\
History of GERD & $8(10.5 \%)$ & $14(18.9 \%)$ & 0.171 \\
History of T2DM & $19(25.0 \%)$ & $15(20.3 \%)$ & 0.560 \\
History of hypertension & $19(25.0 \%)$ & $13(17.6 \%)$ & 0.321 \\
History of dyslipidemia & $11(14.5 \%)$ & $14(18.9 \%)$ & 0.516 \\
History of COPD & $7(9.2 \%)$ & $0(0.0 \%)$ & $0.014 *$ \\
History of OSAS & $5(6.6 \%)$ & $9(12.2 \%)$ & 0.273 \\
\hline
\end{tabular}

$L S G$ laparoscopic sleeve gastrectomy, $L R Y G B$ laparoscopic Roux-en-Y gastric bypass, $B M I$ body mass index, GERD gastroesophageal reflux disease, T2DM type 2 diabetes mellitus, $C O P D$ chronic obstructive pulmonary disease, $O S A S$ obstructive sleep apnea syndrome

${ }^{*} p \leq 0.05 ; * * p \leq 0.01 ; * * * p \leq 0.001$

${ }^{\text {a }}$ Independent samples $t$ test or Fisher's exact test 
Table 2 Differences in quality of life before surgery, 2 months after surgery, and 12 months after surgery

\begin{tabular}{|c|c|c|c|c|c|}
\hline & LSG & Number & LRYGB & Number & $p$ value $^{\mathrm{a}}$ \\
\hline \multicolumn{6}{|l|}{ GerdQ $^{b}$} \\
\hline Baseline & $6.17( \pm 1.53)$ & 71 & $6.44( \pm 1.62)$ & 68 & 0.421 \\
\hline 2 months & $6.95( \pm 2.14)$ & 64 & $5.50( \pm 1.49)$ & 62 & $<.001 * * *$ \\
\hline 12 months & $6.63( \pm 2.26)$ & 63 & $5.60( \pm 1.07)$ & 56 & $0.001 * * *$ \\
\hline \multicolumn{6}{|l|}{ BAROS $^{\mathrm{c}}$} \\
\hline Baseline & $0.54( \pm 1.10)$ & 76 & $0.56( \pm 1.18)$ & 74 & 0.789 \\
\hline 2 months & $1.36( \pm 1.11)$ & 72 & $1.35( \pm 1.02)$ & 63 & 0.958 \\
\hline 12 months & $1.60( \pm 1.29)$ & 68 & $1.82( \pm 0.98)$ & 59 & 0.473 \\
\hline \multicolumn{6}{|l|}{$\mathrm{GIQLI}^{\mathrm{c}}$} \\
\hline Baseline & $112.43( \pm 16.44)$ & 75 & $110.69( \pm 17.51)$ & 72 & 0.587 \\
\hline 2 months & $115.67( \pm 18.90)$ & 71 & $113.84( \pm 18.45)$ & 62 & 0.491 \\
\hline 12 months & $116.71( \pm 19.76)$ & 69 & $118.41( \pm 14.69)$ & 58 & 0.858 \\
\hline \multicolumn{6}{|c|}{ SF-36 Physical Health ${ }^{c}$} \\
\hline Baseline & $65.81( \pm 16.82)$ & 75 & $61.09( \pm 19.58)$ & 73 & 0.178 \\
\hline 2 months & $73.89( \pm 17.84)$ & 71 & $74.68( \pm 16.68)$ & 63 & 0.768 \\
\hline 12 months & $79.97( \pm 13.44)$ & 69 & $79.33( \pm 13.12)$ & 59 & 0.488 \\
\hline \multicolumn{6}{|c|}{ SF-36 Mental Health ${ }^{c}$} \\
\hline Baseline & $73.07( \pm 14.74)$ & 75 & $69.14( \pm 18.85)$ & 73 & 0.234 \\
\hline 2 months & $77.66( \pm 17.87)$ & 71 & $79.88( \pm 14.77)$ & 63 & 0.635 \\
\hline 12 months & $80.27( \pm 16.39)$ & 69 & $79.65( \pm 15.78)$ & 59 & 0.778 \\
\hline \multicolumn{6}{|c|}{ SF-36 Health Change ${ }^{c}$} \\
\hline Baseline & $41.33( \pm 19.49)$ & 75 & $46.92( \pm 23.55)$ & 73 & 0.180 \\
\hline 2 months & $81.34( \pm 22.25)$ & 71 & $81.35( \pm 21.05)$ & 63 & 0.906 \\
\hline 12 months & $93.48( \pm 14.64)$ & 69 & $90.25( \pm 20.24)$ & 59 & 0.540 \\
\hline \multicolumn{6}{|l|}{ EQ-5D ${ }^{c}$} \\
\hline Baseline & $0.73( \pm 0.22)$ & 75 & $0.75( \pm 0.23)$ & 73 & 0.512 \\
\hline 2 months & $0.88( \pm 0.15)$ & 71 & $0.87( \pm 0.18)$ & 63 & 0.790 \\
\hline 12 months & $0.87( \pm 0.17)$ & 69 & $0.87( \pm 0.16)$ & 58 & 0.697 \\
\hline \multicolumn{6}{|l|}{$\mathrm{ACQ}^{\mathrm{b}}$} \\
\hline Baseline & $2.15( \pm 4.74)$ & 74 & $1.67( \pm 3.49)$ & 72 & 0.457 \\
\hline 2 months & $1.37( \pm 2.75)$ & 71 & $0.98( \pm 3.11)$ & 63 & 0.124 \\
\hline 12 months & $1.54( \pm 3.98)$ & 68 & $0.71( \pm 2.07)$ & 58 & 0.307 \\
\hline \multicolumn{6}{|l|}{ DSEQ $^{\mathrm{b}}$} \\
\hline Baseline & $18.72( \pm 5.13)$ & 68 & $19.18( \pm 4.79)$ & 68 & 0.417 \\
\hline 2 months & $16.64( \pm 4.63)$ & 66 & $15.31( \pm 2.62)$ & 58 & 0.430 \\
\hline 12 months & $18.45( \pm 4.63)$ & 66 & $18.28( \pm 4.28)$ & 57 & 0.935 \\
\hline
\end{tabular}

All values are mean $( \pm \mathrm{SD})$

$L S G$ laparoscopic sleeve gastrectomy, $L R Y G B$ laparoscopic Roux-en-Y gastric bypass, GerdQ Gastroesophageal Reflux Disease Questionnaire, BAROS Bariatric Analysis and Reporting Outcome System, GIQLI Gastrointestinal Quality of Life Index, $S F-36$ Short Form (36) Health Survey, EQ-5D EuroQol 5 Dimensions self-report questionnaire, $A C Q$ Asthma Control Questionnaire, $D S E Q$ Dutch Sweet Eating Questionnaire

$* p \leq 0.05 ; * * p \leq 0.01 ; * * * p \leq 0.001$

${ }^{\text {a }}$ Mann-Whitney $U$ test

${ }^{\mathrm{b}}$ A lower score means an improvement in quality of life

${ }^{\mathrm{c}}$ A higher score means an improvement in quality of life of that specific question in their respective group (LSG or LRYGB). This was only done when less than a third of the questions were missing. It has been shown that even when substituting $30 \%$ of a questionnaire by the question's mean, median, or mode, a substantial level of agreement could be achieved [45]. We then analyzed both the original dataset and the dataset in which imputation was used to generate more cases. 


\section{Results}

\section{Patient Characteristics}

A total of 150 patients were included and randomized to either LSG (76 patients) or LRYGB (74 patients). Figure 1 is a flowchart showing the randomization as well as the actual treatment. All patients who underwent surgery were seen after 1 year of follow-up; 128 had returned the 1-year follow-up questionnaires at the time of analysis. Because of missing data, the amount of patients of which any follow-up moment was complete differed per questionnaire, ranging from 131 to 148 patients at baseline and 119 to 128 patients at 1-year follow-up. By substituting missing values with the question's median or mode, we were able to add up to 15 extra cases per questionnaire to be analyzed. Both the original data and the substituted data were analyzed, and results did not differ significantly before and after imputation.

Table 1 shows that characteristics of both groups were similar at baseline, except that the LSG group contained significantly more people with COPD.

\section{Quality of Life}

Comparisons of postoperative questionnaire scores between the LSG group and the LRYGB group are shown in Table 2. Two months after surgery, the mean GerdQ score was $6.95 \pm 2.14$ in the LSG group versus $5.50 \pm 1.49$ in the LRYGB group $(p<0.001)$. After 1 year, the LSG group and the LRYGB group had a mean GerdQ score of $6.63 \pm 2.26$ and $5.60 \pm 1.07$, respectively $(p=0.001)$. No other postoperative questionnaires showed significant differences.

Table 3 shows the mean differences between baseline scores and scores after 2 and after 12 months within each group. Most QOL questionnaires showed significant improvement in scores between baseline and after 12 months. The GerdQ score deteriorated in the LSG group after 2 months, but recovered after 1 year. Figure 2 is a graphical representation of the results shown in Tables 2 and 3.

\section{Weight Loss and Complications}

Table 4 shows BMI at baseline, after 2 months, and after 12 months (see also Fig. 3), as well as \%EWL after 2 months and after 12 months. It also includes the surgical complication rate for the first year after surgery. There were no differences in BMI or weight loss. The complication rate was $6.6 \%$ in LSG and $10.8 \%$ in LRYGB $(p=0.398)$. Both groups had one anastomotic leakage. There were two readmissions within 30 days in each group. There were no reported surgical site infections, and there was no mortality.
Table 3 Improvement in quality of life over time

\begin{tabular}{|c|c|c|c|c|}
\hline & LSG & $p$ value $^{\mathrm{a}}$ & LRYGB & $p$ value $^{\mathrm{a}}$ \\
\hline \multicolumn{5}{|l|}{ GerdQ $^{b}$} \\
\hline Baseline vs 2 months & 0.78 & $0.045^{*}$ & -0.94 & $<.001 * * *$ \\
\hline Baseline vs 12 months & 0.46 & 0.132 & -0.84 & $0.001 * * *$ \\
\hline \multicolumn{5}{|l|}{ BAROS $^{c}$} \\
\hline Baseline vs 2 months & 0.82 & $<.001 * * *$ & 0.79 & $<.001 * * *$ \\
\hline Baseline vs 12 months & 1.06 & $<.001 * * *$ & 1.26 & $<.001 * * *$ \\
\hline \multicolumn{5}{|l|}{ GIQLI $^{\mathrm{c}}$} \\
\hline Baseline vs 2 months & 3.24 & $0.039 *$ & 3.15 & 0.075 \\
\hline Baseline vs 12 months & 4.28 & $0.012 *$ & 7.72 & $0.003 * *$ \\
\hline \multicolumn{5}{|l|}{ SF-36 Physical Health ${ }^{c}$} \\
\hline Baseline vs 2 months & 8.08 & $<.001 * * *$ & 13.59 & $<.001 * * *$ \\
\hline Baseline vs 12 months & 14.16 & $<.001 * * *$ & 18.24 & $<.001 * * *$ \\
\hline \multicolumn{5}{|l|}{ SF-36 Mental Health ${ }^{c}$} \\
\hline Baseline vs 2 months & 4.59 & $0.008 * *$ & 10.74 & $<.001 * * *$ \\
\hline Baseline vs 12 months & 7.20 & $<.001 * * *$ & 10.51 & $<.001 * * *$ \\
\hline \multicolumn{5}{|l|}{ SF-36 Health Change ${ }^{c}$} \\
\hline Baseline vs 2 months & 40.01 & $<.001 * * *$ & 34.43 & $<.001 * * *$ \\
\hline Baseline vs 12 months & 52.15 & $<.001 * * *$ & 43.33 & $<.001 * * *$ \\
\hline \multicolumn{5}{|l|}{$E Q-5 D^{c}$} \\
\hline Baseline vs 2 months & 0.15 & $<.001 * * *$ & 0.12 & $<.001 * * *$ \\
\hline Baseline vs 12 months & 0.14 & $<.001 * * *$ & 0.12 & $<.001 * * *$ \\
\hline \multicolumn{5}{|l|}{$\mathrm{ACQ}^{\mathrm{b}}$} \\
\hline Baseline vs 2 months & -0.78 & 0.148 & -0.69 & $0.014 *$ \\
\hline Baseline vs 12 months & -0.61 & 0.105 & -0.86 & $0.001 * *$ \\
\hline \multicolumn{5}{|l|}{ DSEQ $^{\mathrm{b}}$} \\
\hline Baseline vs 2 months & -2.08 & $0.015^{*}$ & -3.87 & $<.001 * * *$ \\
\hline Baseline vs 12 months & -0.27 & 0.753 & -0.9 & 0.354 \\
\hline
\end{tabular}

All values are mean differences

$L S G$ Laparoscopic sleeve gastrectomy, LRYGB Laparoscopic Roux-en-Y gastric bypass, GerdQ Gastroesophageal Reflux Disease Questionnaire, BAROS Bariatric Analysis and Reporting Outcome System, GIQLI Gastrointestinal Quality of Life Index, $S F-36$ Short Form (36) Health Survey, $E Q-5 D$ EuroQol 5 Dimensions self-report questionnaire, $A C Q$ Asthma Control Questionnaire, DSEQ Dutch Sweet Eating Questionnaire ${ }^{*} p \leq 0.05 ; * * p \leq 0.01 ; * * p \leq 0.001$

${ }^{a}$ Wilcoxon's signed rank test

${ }^{\mathrm{b}}$ A lower score means an improvement in quality of life

${ }^{\mathrm{c}}$ A higher score means an improvement in quality of life

\section{Discussion}

This trial has shown that patients after both LSG and LRYGB had overall comparable improvement in QOL after 1 year of follow-up. The only significant difference was that patients in the LSG group had a higher mean GerdQ score after 1 year, meaning they had more symptoms of GERD. A remarkable finding was that people in the LSG group had increased GerdQ scores after surgery, while patients in the LRYGB 
a GerdQ

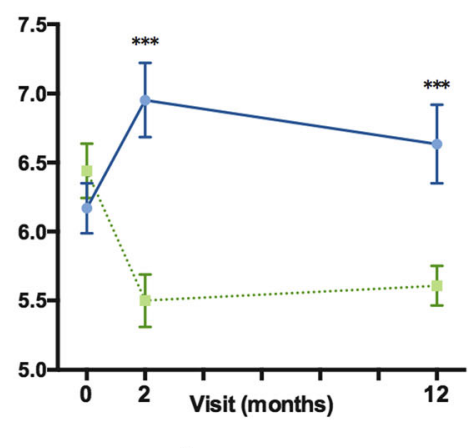

d SF-36 Physical Health

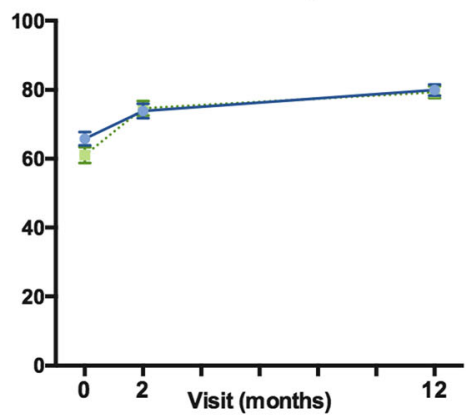

g EQ-5D

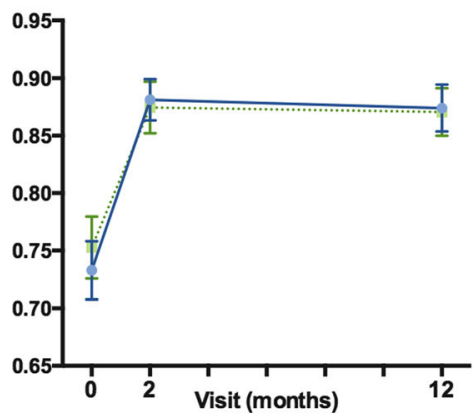

Fig. 2 a-i Improvement in quality of life over time. All values are mean, whiskers show standard error of the mean (SEM). LSG laparoscopic sleeve gastrectomy, $L R Y G B$ laparoscopic Roux-en-Y gastric bypass, GerdQ Gastroesophageal Reflux Disease Questionnaire, BAROS

group had significantly decreased GerdQ scores after surgery. The \%EWL was equal in both groups, so it is unlikely that this has influenced the difference in GERD symptoms.

In this trial, GERD was not objectified by $\mathrm{pH}$ monitoring, the gold standard to diagnose GERD, nor by gastroscopy; therefore, we can only rely on GerdQ scores. Despite the significantly worse GerdQ scores in the LSG group, overall quality of life was not compromised, as shown by similar increases in SF-36, EQ-5D, BAROS, and GIQLI scores in both groups. This raises the question whether a slightly higher GerdQ score is of clinical importance.

Other studies have described similar results regarding GERD after LSG. One study concluded that LSG was associated with the development of GERD (diagnosed by using 24-h $\mathrm{pH}$ monitoring) in two thirds of patients who were asymptomatic before surgery [46]. Another study that also used $\mathrm{pH}$
C GIQLI

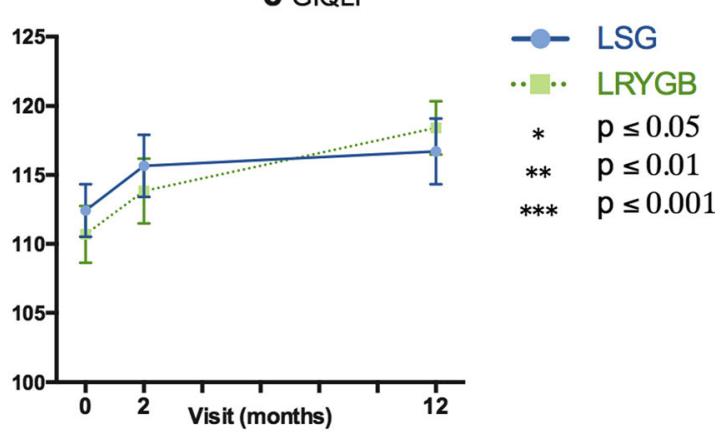

f SF-36 Health Change

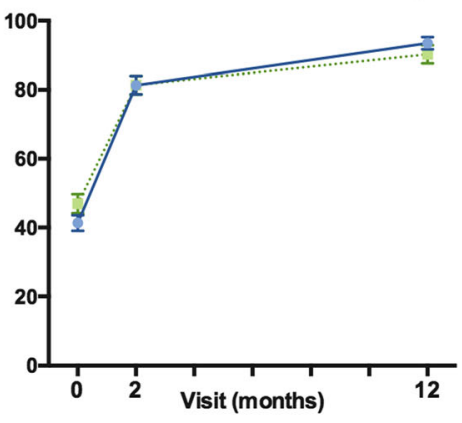

i DSEQ

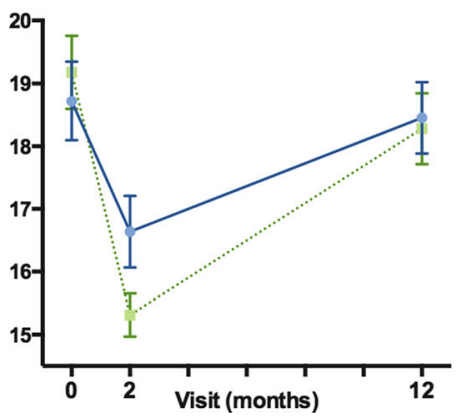

Bariatric Analysis and Reporting Outcome System, GIQLI Gastrointestinal Quality of Life Index, $S F-36$ Short Form (36) Health Survey, $E Q-5 D$ EuroQol 5 Dimensions self-report questionnaire, $A C Q$ Asthma Control Questionnaire, DSEQ Dutch Sweet Eating Questionnaire

monitoring found that over $80 \%$ of previously asymptomatic patients suffered from GERD 1 year after LSG [47]. A third study diagnosed GERD using a combination of symptom scoring and/or objective evidence such as contrast studies, gastroscopy, and $\mathrm{pH}$ monitoring. They found that $3 \%$ of all patients developed de novo GERD after LSG but that almost all patients responded well to treatment with a PPI [48]. Other studies that have used either symptom questionnaires or gastroscopy found similar results regarding the need for PPIs after LSG [49, 50]. One more study found not only that $7 \%$ of patients who were asymptomatic before surgery developed de novo GERD after LSG but also that symptoms resolved in $96 \%$ of patients who had GERD preoperatively [27]. Another study has found that patients with GERD after LSG had a significantly higher rate of readmission within 30 days, but the odds ratio for converting LSG into LRYGB was only 
Table 4 Body mass index, excess weight loss, and complications

\begin{tabular}{lccccc}
\hline & LSG & Number & LRYGB & Number & $p$ value $^{\mathrm{a}}$ \\
\hline BMI & & & & \\
$\quad$ Baseline & $44.17( \pm 5.00)$ & 76 & $44.03( \pm 5.28)$ & 73 & 0.860 \\
2 months & $37.53( \pm 5.09)$ & 72 & $39.45( \pm 1291)$ & 66 & 0.261 \\
12 months & $30.77( \pm 5.70)$ & 70 & $30.18( \pm 4.63)$ & 60 & 0.516 \\
$\%$ EWL & & & $33.16( \pm 9.45)$ & 66 & 0.158 \\
2 months & $36.03( \pm 14.07)$ & 72 & $75.60( \pm 21.02)$ & 60 & 0.537 \\
12 months & $73.21( \pm 22.82)$ & 70 & $8(10.8 \%)$ & 74 & 0.398 \\
Complications & $5(6.6 \%)$ & 76 & &
\end{tabular}

All values are mean $( \pm \mathrm{SD})$

$L S G$ laparoscopic sleeve gastrectomy, $L R Y G B$ laparoscopic Roux-en-Y gastric bypass, $B M I$ body mass index, $\% E W L$ percentage excess weight loss

$* p \leq 0.05 ; * * p \leq 0.01 ; * * * \leq 0.001$

${ }^{a}$ Independent samples $t$ test or Fisher's exact test higher in Caucasian patients [51]. Finally, the results of a recently published randomized controlled trial comparing LSG with LRYGB show that weight loss, quality of life, and complications did not differ significantly after 3 years. However, there was a slight difference in remission of GERD in favor of the LRYGB group [52]. Another recent systematic review and meta-analysis remained inconclusive on the subject, stating the heterogeneity of recent publications and their paradoxical outcomes [17]. Therefore, from the currently available literature, we can conclude that there is no clear evidence that pre-existing GERD should be an absolute contraindication for LSG.

Some studies have tried to come up with solutions to minimize GERD after LSG. A case series of 40 patients has shown that $95 \%$ of patients with pre-existing GERD experienced complete remission when anterior fundoplication with a posterior crural approximation was added to LSG. During stapling of the sleeve, a small fundus is left to use for anterior

\section{Body Mass Index (BMI)}

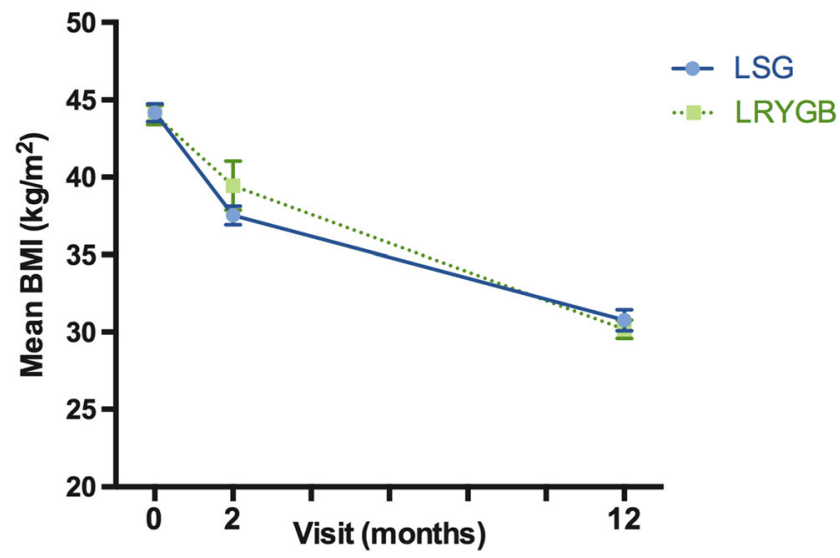

Fig. 3 Body mass index over time. BMI body mass index, $L S G$ laparoscopic sleeve gastrectomy, $L R Y G B$ laparoscopic Roux-en-Y gastric bypass fundoplication [53]. Another study used a similar technique of fundoplication and called it the Nissen Sleeve (N-Sleeve), after the Nissen fundoplication. They found that $88 \%$ of patients who had pre-existent esophageal syndromes had experienced remission 1 year after N-Sleeve [54].

The main limitation of the current trial is the small sample size. Even though 150 patients seemed like a good sample size, the analyses had to be done with substantially less data because of patients skipping or forgetting questions from the questionnaires. The authors tried to counter this by using median or mode imputation on the missing data. On top of the missing questions, there were still some patients who had not returned the 1-year follow-up questionnaires. Other limitations include the lack of data on actual PPI usage and the lack of esophageal $\mathrm{pH}$ monitoring for diagnosing GERD.

We recommend that further research should be focused on the implications of GERD after LSG, rather than the mere presence of symptoms. As this trial and some other studies have already shown, a lot of patients did not suffer from these symptoms and reported good quality of life on other validated questionnaires. Also, the use of a PPI could be a simple solution to many patients with GERD symptoms. Future studies should also evaluate other options to counter GERD after LSG, like anterior fundoplication with posterior crural approximation or the $\mathrm{N}$-Sleeve.

Acknowledgments We would like to thank Irene Friskes, bariatric physician assistant at the Fransicus Gasthuis, for her help in gathering and organizing the filled out questionnaires, as well as for her help with creating and maintaining the study database.

Funding This study was not funded by any grants.

\section{Compliance with Ethical Standards}

Conflict of Interest The authors declare that they have no conflict of interest. 
Ethical Approval All procedures performed in studies involving human participants were in accordance with the ethical standards of the institutional and/or national research committee and with the 1964 Helsinki declaration and its later amendments or comparable ethical standards.

Informed Consent Informed consent was obtained from all individual participants included in the study.

\section{References}

1. CBS StatLine, updated 5-4-2016.

2. Calle EE, Thun MJ, Petrelli JM, et al. Body-mass index and mortality in a prospective cohort of U.S. adults. N Engl J Med. 1999;15: 1097-105.

3. Corley DA, Kubo A. Body mass index and gastroesophageal reflux disease: a systematic review and meta-analysis. Am J Gastroenterol. 2006;108:2619-28.

4. Elte JWF, Castro Cabezas M, Vrijland WW, et al. Proposal for a multidisciplinary approach to the patient with morbid obesity: the Sint Franciscus Hospital morbid obesity program. Eur J Intern Med. 2008;19:92-8.

5. Gloy VL, Briel M, Bhatt DL, et al. Bariatric surgery versus nonsurgical treatment for obesity: a systemic review and meta-analysis of randomized controlled trials. BMJ. 2013;347:f5934.

6. James PT, Rigby N, Leach R, et al. The obesity epidemic, metabolic syndrome and future prevention strategies. Eur J Carddiovasc Prev Rehab. 2004;11:3-8.

7. Maggard MA, Shugarman LR, Suttorp M, et al. Meta-analysis: surgical treatment of obesity. Ann Intern Med. 2005;142:547-59.

8. Sjöström L, Narbro K, Sjöström CD, et al. Effects of bariatric surgery on mortality in Swedish obese subjects. N Engl J Med. 2007;357:741-52.

9. Van Huisstede A, Rudolphus A, Castro Cabezas M, et al. Effect of bariatric surgery on asthma control, lung function and bronchial and systemic inflammation in morbidly obese subjects with asthma. Thorax. 2015;70(7):659-67.

10. Buchwald H, Avidor Y, Braunwald E, et al. Bariatric surgery: a systematic review and meta-analysis. JAMA. 2004;292:1724-37.

11. Buchwald H, Oien DM. Metabolic/bariatric surgery worldwide 2011. Obes Surg. 2013;4:427-36.

12. Angrisani L, Santonicola A, Iovino P, et al. Bariatric surgery worldwide 2013. Obes Surg. 2015;25(10):1822-32.

13. Gjessing HR, Nielsen HJ, Mellgren G, et al. Energy intake, nutritional status and weight reduction in patients one year after laparoscopic sleeve gastrectomy. Spring. 2013;2:352.

14. Tack J, Arts J, Caenepeel P, et al. Pathophysiology, diagnosis and management of postoperative dumping syndrome. Nat Rev Gastroenterol Hepatol. 2009;6:583-90.

15. Loss AB, de Souza AA, Pitombo CA, et al. Analysis of the dumping syndrome on morbid obese patients submitted to Roux en $\mathrm{Y}$ gastric bypass. Rev Col Bras Cir. 2009;36:413-9.

16. Dalcanale L, Oliveira CP, Faintuch J, et al. Long-term nutritional outcome after gastric bypass. Obes Surg. 2010;20:181-7.

17. Oor JE, Roks DJ, Ünlü Ç, et al. Laparoscopic sleeve gastrectomy and gastroesophageal reflux disease: a systematic review and metaanalysis. Am J Surg. 2016;211:250-67.

18. Peterli R, Borbely Y, Kern B, et al. Early results of the Swiss Multicentre Bypass or Sleeve Study (SM-BOSS). Ann Surg. 2013;258(5):690-5.

19. Karamanakos SN, Vagenas K, Kalfarentzos F, et al. Weight loss, appetite suppression, and changes in fasting and postprandial ghrelin and peptide-YY levels after Roux-en-Y gastric bypass and sleeve gastrectomy: a prospective, double blind study. Ann Surg. 2008;247:401-7.

20. Kehagias I, Karamanakos SN, Argentou M, et al. Randomized clinical trial of laparoscopic Roux-en-Y gastric bypass versus laparoscopic sleeve gastrectomy for the management of patients with BMI $<50 \mathrm{~kg} / \mathrm{m} 2$. Obes Surg. 2011;11:1650-6.

21. Lee WJ, Chong K, Ser KH, et al. Gastric bypass vs sleeve gastrectomy for type 2 diabetes mellitus: a randomized controlled trial. Arch Surg. 2011;146:143-8.

22. Schauer PR, Kashyap SR, Wolski K, et al. Bariatric surgery versus intensive medical therapy in obese patients with diabetes. N Engl J Med. 2012;366:1567-76.

23. Helmiö M, Victorzon M, Ovaska J, et al. Comparison of short-term outcome of laparoscopic sleeve gastrectomy and gastric bypass in the treatment of morbid obesity: a prospective randomized controlled multicenter SLEEVEPASS study with 6-month follow up. Scand J Surg. 2014;103(3):175-81.

24. Hong JS, Kim WW, Han SM. Five-year results of laparoscopic sleeve gastrectomy in Korean patients with lower body mass index. Obes Surg. 2015;25(5):824-9.

25. Felsenreich DM, Langer FB, Kefurt R, et al. Weight loss, weight regain, and conversions to Roux-en-Y gastric bypass: 10-year results of laparoscopic sleeve gastrectomy. Surg Obes Relat Dis. 2016 Nov;12(9):1655-62.

26. Himpens J, Dobbeleir J, Peeters G. Long-term results of laparoscopic sleeve gastrectomy for obesity. Ann Surg. 2010;252(2): 319-24.

27. Gadiot RP, Biter LU, van Mil S, et al. Long-term results of laparoscopic sleeve gastrectomy for morbid obesity: 5 to 8 -year results. Obes Surg. 2017 Jan;27(1):59-63.

28. Overs SE, Freeman RA, Zarshenas N, et al. Food tolerance and gastrointestinal quality of life following three bariatric procedures: adjustable gastric banding, Roux-en-Y gastric bypass and sleeve gastrectomy. Obes Surg. 2012;22:536-43.

29. Lee WJ, Pok EH, Almulaifi A, et al. Medium-term results of laparoscopic sleeve gastrectomy: a matched comparison with gastric bypass. Obes Surg. 2015;25:1431-8.

30. Major P, Matlok M, Pedziwiatr M, et al. Quality of life after bariatric surgery. Obes Surg. 2015;25:1703-10.

31. Zhang $\mathrm{Y}$, Zhao $\mathrm{H}$, Cao Z, et al. A randomized clinical trial of laparoscopic Roux-en-Y gastric bypass and sleeve gastrectomy for the treatment of morbid obesity in China: a 5-year outcome. Obes Surg. 2014;24:1617-24.

32. International Federation for the Surgery of Obesity and Metabolic Disorders (IFSO). Are You a Candidate? Selection criteria for bariatric or weight loss surgery. Available from: http://www.ifso.com/ are-you-a-candidate/. Accessed 4 Jan 2017.

33. Fornari F, Madalosso CA, Farre R, et al. The role of gastrooesophageal pressure gradient and sliding hiatal hernia on pathological gastro-oesophageal reflux in severely obese patients. Eur J Gastroenterol Hepatol. 2010;22:404-11.

34. Van den Heuvel M, Horchner R, Wijtsma A, et al. Sweet eating: a definition and development of the Dutch Sweet Eating Questionnaire. Obes Surg. 2011;6:714-21.

35. Biter LU, Gadiot RP, Grotenhuis BA, et al. The Sleeve Bypass Trial: a multicentre randomized controlled trial comparing the long term outcome of laparoscopic sleeve gastrectomy and gastric bypass for morbid obesity in terms of excess BMI loss percentage and quality of life. BMC Obes. 2015;2:30.

36. Gadiot RP, Biter LU, Zengerink HJ, et al. Laparoscopic sleeve gastrectomy with an extensive posterior mobilization: technique and preliminary results. Obes Surg. 2012;2:320-9.

37. Geubbels N, Bruin SC, Acherman YI, et al. Fast track care for gastric bypass patients decreases length of stay without increasing complications in an unselected patient cohort. Obes Surg. 2014;24(3):390-6. 
38. Clavien PA, Barkun J, de Oliveira ML, et al. The Clavien-Dindo classification of surgical complications: five-year experience. Ann Surg. 2009;250(2):187-96.

39. Eypasch E, Williams JI, Wood-Dauphinee S, et al. Gastrointestinal Quality of Life Index: development, validation and application of a new instrument. Br J Surg. 1995;82:216-22.

40. Oria HE, Moorehead MK. Bariatric Analysis and Reporting Outcome System (BAROS). Obes Surg. 1998;8(5):487-99.

41. Jones R, Junghard O, Dent J, et al. Development of the GerdQ, a tool for the diagnosis and management of gastro-oesophageal reflux disease in primary care. Aliment Pharmacol Ther. 2009;30:1030-8.

42. Juniper EF, O’Byrne PM, Guyatt GH, et al. Development and validation of a questionnaire to measure asthma control. Eur Respir J. 1999;14:902-7.

43. Lamers LM, Stalmeier PF, McDonnell J, et al. Measuring the quality of life in economic evaluations: the Dutch EQ-5D tariff. [Article in Dutch] Ned Tijdschr Geneeskd. 2005;149(28):1574-8.

44. Hays RD, Sherbourne CD, Mazel RM. The RAND 36-Item Health Survey 1.0. Health Econ. 1993;2:217-27.

45. Shrive FM, Stuart H, Quan H, et al. Dealing with missing data in a multi-question depression scale: a comparison of imputation methods. BMC Med Res Methodol. 2006;6:57.

46. Thereaux J, Barsamian $\mathrm{C}$, Bretault $\mathrm{M}$, et al. $\mathrm{pH}$ monitoring of gastro-oesophageal reflux before and after laparoscopic sleeve gastrectomy. Brit J Surg. 2016;103:399-406.

47. Georgia D, Stamatina T, Maria N, et al. 24-h multichannel intraluminal impedance PH-metry 1 year after laparoscopic sleeve gastrectomy: an objective assessment of gastroesophageal reflux disease. Obes Surg. 2017 Mar;27(3):749-53.

48. Hendricks L, Alvarenga E, Dhanabalsamy N, et al. Impact of sleeve gastrectomy on gastroesophageal reflux disease in a morbidly obese population undergoing bariatric surgery. Surg Obes Relat Dis. 2016;12(3):511-7.

49. Arman GA, Himpens J, Dhaenens J, et al. Long-term (11+ years) outcomes in weight, patient satisfaction, comorbidities, and gastroesophageal reflux treatment after laparoscopic sleeve gastrectomy. Surg Obes Relat Dis. 2016 Dec;12(10):1778-86.

50. Barr AC, Frelich MJ, Bosler ME, et al. GERD and acid reduction medication use following gastric bypass and sleeve gastrectomy. Surg Endosc. 2017 Jan;31(1):410-5.

51. Obeid T, Krishnan A, Abdalla G, et al. GERD is associated with higher long-term reoperation rates after bariatric surgery. J Gastrointest Surg. 2016;20:119-24.

52. Peterli R, Wölnerhanssen BK, Vetter D, et al. Laparoscopic sleeve gastrectomy versus Roux-Y-gastric bypass for morbid obesity-3year outcomes of the prospective randomized Swiss Multicenter Bypass Or Sleeve Study (SM-BOSS). Ann Surg. 2017;265:46673.

53. Hawasli A, Reyes M, Hare B, et al. Can morbidly obese patients with reflux be offered laparoscopic sleeve gastrectomy? A case report of 40 patients. Am J Surg. 2016;211(3):571-6.

54. Nocca D, Skalli EM, Boulay E, et al. Nissen Sleeve (N-Sleeve) operation: preliminary results of a pilot study. Surg Obes Relat Dis. 2016 Dec;12(10):1832-7. 